\title{
Cine-Foro como estrategia Didáctica para el desarrollo del Pensamiento Crítico
}

\author{
Jhon Fredy Mejia Vargas * \\ Angie Stefanny Pineda Infante ${ }^{* * *}$
}

Artículo de investigación

Fecha de Recepción: 7 diciembre 2017.

Fecha de Aprobación: 30 abril 2018.

\section{Resumen}

El siguiente artículo muestra los resultados parciales de una investigación que se ha propuesto verificar los efectos del Cine-Foro como estrategia didáctica para el desarrollo de habilidades de Pensamiento Crítico en una población de estudiantes de Sexto grado. Se ha tomado la Investigación Acción como la metodología más adecuada a los requerimientos de la investigación, el enfoque metodológico es mixto con énfasis en lo cualitativo.

Se trabajó el concepto de Pensamiento Crítico desde la postura intelectual de Richard Paul y \& Elder (2003). Desde este punto de vista el pensamiento crítico se compone de ocho elementos, a saber: Propósito, Problema, Información, Concepto, Supuestos, Inferencias, Consecuencias y Puntos de vista.

Los instrumentos utilizados para la recolección de la información fueron, en primer lugar, la prueba Watson y Glaser, una prueba diagnóstica $(50$ preguntas). Después se implementó la estrategia del Cine-Foro (Cañizalez \& Pulido, 2015) dividido en tres ciclos de películas, cada ciclo se evaluó con un
* Escuela Normal Superior Antonia Santos -SantanderColombia jhonmejia963@gmail. com

** Escuela Normal Superior Antonia Santos Santander-Colombia angiespineda@gmail.com

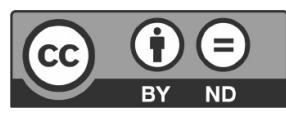


cuestionario de 8 preguntas y un taller de 24 preguntas, los dos instrumentos evalúan los elementos del pensamiento por medio de estándares intelectuales. Aquí se presentan los resultados de la prueba diagnóstica, del cuestionario \#1 y del taller \#1, correspondientes al primer ciclo. Al comparar estos datos se pudo corroborar el éxito parcial de la intervención.

Palabras Clave: Pensamiento Crítico, Elementos del Pensamiento, Estándares Intelectuales, Didáctica, Cine-Foro, Ciencias Sociales. 


\section{Introducción}

Este proyecto tiene como propósito determinar los efectos de la estrategia del Cine-Foro sobre el pensamiento crítico en estudiantes del grado sexto B de la ENSAS, a partir del desglose de los elementos del pensamiento: propósito, problema, deducción, información, conceptos, supuestos, consecuencias y puntos de vista, propuestos por Richard Paul y Linda Elder.

La investigación muestra una de las maneras en que el Cine-Foro se convierte en un espacio vital para la construcción de pensamiento crítico (P.C), se impone la necesidad de encontrar estrategias para el desarrollo de procesos de pensamiento crítico al interior de la Escuela Normal Superior Antonia Santos de Puente Nacional. El Cine-Foro es la estrategia escogida para crear con la población estudiantil un ambiente ideal para la expresión de la reflexión crítica.

Paul \& Elder afirman que la única capacidad que podemos usar para aprender, es el pensamiento humano. Si pensamos bien mientras aprendemos, aprendemos bien. Si pensamos mal mientras aprendemos, aprendemos mal. (2005, p. 10). Por consiguiente, muchas de las dificultades que son típicas de los procesos de enseñanza aprendizaje se derivan de inadecuados procedimientos del pensamiento.

A menudo estos fallos están relacionados con predisposiciones naturales de los individuos que subyacen a sus comportamientos y limitan sus posibilidades de interpretación. En este sentido Herrero (2016) señala que de estas predisposiciones la primera es evitar sentirse marginado y en consecuencia las personas buscan identificarse con el grupo, adaptando sus opiniones a la mayoría; la segunda es dar por sentado que las actitudes personales son universales y todos las comparten y la tercera es el prejuicio, que consiste en dar las cosas por hecho sin verificarlas (p.21). Desde este punto de vista, el individuo percibe que la autonomía es un asunto peligroso que debe evitarse, percibe además que él es como todos y todos son como él, de aquí, que su modo de interpretar se base en prejuicios y no en la razón.

Tales predisposiciones indudablemente afectan los resultados de cualquier proyecto educativo, entorpeciendo de paso la posibilidad de desarrollar un auténtico pensamiento crítico. La educación lamentablemente suele adolecer de estas mismas predisposiciones. En palabras de Paul, et al., (1990, p. 4), hay que considerar cómo los docentes generalmente se limitan a una enseñanza factual, pues continuamente a los estudiantes se les bombardea con hechos fácilmente retenibles (por ejemplo, las principales exportaciones de un país extranjero), y simplemente se espera que los retengan, pero los estudiantes no entienden claramente porqué deberían recordar estos hechos. Esto es lo que se llama educación tradicional, una enseñanza enfocada en la transmisión pasiva de información y datos.

Los autores advierten, que "diariamente nos enfrentamos con un exceso
Este proyecto tiene como propósito determinar los efectos de la estrategia del Cine-Foro sobre el pensamiento crítico en estudiantes del grado sexto B de la ENSAS, a partir del desglose de los elementos del pensamiento 


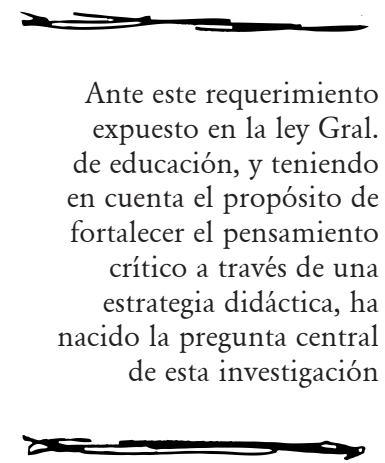

de información, y mucha de esa información ha sido artificiosamente envuelta para servir a grupos con intereses personales y no al ciudadano en particular o al bien común" (Paul \& Elder, 2005, p. 12). De manera que el problema no yace solamente en la información como tal, sino que el verdadero peligro radica en que en gran parte de las relaciones humanas median intereses egoístas que buscan la manipulación y el dominio sobre los otros. Es así que una actitud acrítica no solamente compromete las posibilidades de aprendizaje de los individuos, sino que además los convierte en entes fácilmente manejables, aún en detrimento de su propia integridad.

Es necesario tener en cuenta, además, que la ley General de Educación 115 de 1994 de Colombia reconoce que la educación debe encarar circunstancias cambiantes y debe poder responder a los desafíos que el mundo moderno ofrece. Además, esta ley señala que, un objetivo de la educación colombiana es el fomento de "la capacidad reflexiva y crítica sobre los múltiples aspectos de la realidad y la comprensión de los valores éticos, morales, religiosos y de convivencia en sociedad" (art. 30. Ley 115 de 1994). De igual manera, la presidencia de Colombia en el acto de sanción de la ley de educación de 1994 estableció que:

El país requiere de ciudadanos educados para la democracia, preparados y capacitados para investigar y dar respuestas efectivas a los múltiples problemas de la ciencia, del pensamiento y de la técnica, en un mundo que se trasforma aceleradamente y que impone niveles crecientes de competitividad. (H. Stefenn Prolibros, 2002, p. 3).

Ante este requerimiento expuesto en la ley Gral. de educación, y teniendo en cuenta el propósito de fortalecer el pensamiento crítico a través de una estrategia didáctica, ha nacido la pregunta central de esta investigación; ¿cómo el cine-foro puede empoderar el desarrollo del pensamiento crítico en los estudiantes de sexto grado de la escuela normal superior Antonia Santos de Puente Nacional? Para el desarrollo de la presente investigación se han planteado los siguientes objetivos para atender a la problemática planteada y que se ajustarán a las condiciones de la investigación-acción:

General, Desarrollar procesos de pensamiento crítico a partir del Cineforo como estrategia didáctica en los estudiantes de Sexto B grado de la Escuela Normal Superior Antonia Santos (ENSAS). Específicos, 1). Diagnosticar los niveles de pensamiento crítico en los estudiantes de Sexto B de la ENSAS. 2). Diseñar y aplicar la estrategia didáctica del cine-foro para el desarrollo de procesos de pensamiento crítico. Y 3). Evaluar la efectividad de la estrategia propuesta aplicada.

\section{Ladidácticaylaenseñanza del pensamiento crítico}

Carrasco (2004), define la didáctica como "la dialéctica (que) lleva a la idea de enseñar. El término griego del que deriva, el verbo didaskein, significa 
enseñar, instruir, explicar” (p. 18). Desde este punto de vista, la enseñanza es un asunto eminentemente práctico. Por eso la didáctica juega un papel muy importante en los procesos educativos, puesto que una propuesta de educación alejada de la práctica no puede cumplir sus propósitos pedagógicos.

Partiendo de esta base se puede afirmar que las habilidades de pensamiento identificadas por múltiples autores como propias del PC, como examinar, revisar, analizar, comparar, deducir, entre otras, solo pueden tener un desarrollo efectivo en el contexto de una educación que asuma los procesos de enseñanza-aprendizaje con un enfoque crítico y didáctico. Se hace necesaria una nueva concepción de la educación que oriente hacia las prácticas en el aula de clase y que supere la brecha creada entre contenidos programáticos y experiencia vital.

Ahora, no se puede ignorar que la didáctica, al situarse en un contexto vivencial involucra a los participantes en procesos de enseñanza-aprendizaje y aborda las problemáticas educativas partiendo de ambientes concretos de trabajo. De esta manera la didáctica adquiere una connotación normativa, pues no se limita a la explicación de un determinado fenómeno, sino que fija normas de conducta desde su esfera teórica.

\section{Elementos del pensamiento $y$ Estándares intelectuales}

"El pensamiento crítico presupone el conocimiento de las estructuras más básicas del pensamiento (los elementos del pensamiento) y los estándares intelectuales más básicos del pensamiento (estándares intelectuales universales)" (Paul \& Elder 2005, p. 7). En efecto, por medio del desglose del pensamiento se llega a sus elementos constitutivos; dichos elementos a su vez se encuentran atravesados por criterios o estándares intelectuales que apuntan a la corrección del pensamiento. Estos aspectos del pensamiento son indisolubles, y en su grado óptimo de desarrollo representan el verdadero pensamiento crítico en todas sus dimensiones.

Paul \& Elder (2003) afirman que el pensamiento crítico es un modo de pensar acerca de cualquier tema, contenido o problema, en el cual la persona que piensa mejora la calidad de su pensamiento al apoderarse de las estructuras inherentes al acto de pensar y al someterlas a estándares intelectuales. En efecto, es necesario señalar la importancia que tiene esto para la educación en general, si se enseña a pensar críticamente desde las escuelas y las aulas, este conocimiento podrá ser útil en todos los campos de la experiencia, mientras que la enseñanza que solo atiende a los contenidos académicos sacrifica la flexibilidad necesaria para adaptarse a las condiciones particulares de los estudiantes y para sacar el máximo potencial de ellos.

El pensamiento crítico es el proceso de analizar y evaluar el pensamiento con el propósito de mejorarlo. En otras palabras, cada elemento de pensamiento
Paul \& Elder (2003) afirman que el pensamiento crítico es un modo de pensar acerca de cualquier tema, contenido o problema, en el cual la persona que piensa mejora la calidad de su pensamiento al apoderarse de las estructuras inherentes al acto de pensar y al someterlas a estándares intelectuales. 
clasificado por Paul \& Elder, (2005) como estándares intelectuales, se define por:

- Propósitos, Metas y objetivos compartidos (que orientan),

- Problemas compartidos (cuyas soluciones son procuradas),

- Información y datos compartidos (que usan como bases empíricas)

- Conceptos e ideas especializadas compartidas (que usan para organizar los datos),

- Suposiciones claves compartidas (que les dan un conjunto de puntos comunes para empezar).

- Inferencias (que surgen de los supuestos y conllevan consecuencias)

- Consecuencias e implicaciones, conclusiones (consecuencias extraídas de las inferencias o interpretaciones)

- Un punto de vista compartido (lo que les permite seguir metas comunes dentro de un marco de referencia común). (p. 15).

Los ocho elementos del pensamiento que se han descrito deben además ser evaluados con estándares intelectuales, que para Paul tienen un carácter universal, a saber, Claridad, precisión, profundidad, justicia, amplitud, lógica, coherencia, relevancia; para fines prácticos de esta investigación dicha evaluación contempló la utilización solamente de los estándares de claridad y de precisión, que sirvieron de indicadores. Esta decisión se funda en la propia opinión de Paul y Elder quienes afirman: "Los criterios de claridad y precisión del pensamiento crítico son todo lo que se requiere a nivel de comprensión inicial. Cuando se aplica a la disciplina o a la profesión misma, pueden requerirse la profundidad, amplitud y significancia” (2005, p. 19).

\section{Método Socrático de Interrogación}

El trabajo teórico de Paul y Elder se sustenta en buena medida en el empleo de estrategias inspiradas en el método socrático de interrogación, en sus libros exponen gran parte de su análisis sobre este tópico, en esencia, éste está basado en la idea de que todo pensamiento se basa en una lógica o estructura, que cualquier declaración revela solo parcialmente lo que subyace al pensamiento, expresando nada más que una pequeña parte del sistema de creencias interconectadas del cual son parte. Su propósito es exponer la lógica del pensamiento de alguien. (Paul, et al., 1990).

Según Torres, Arévalo, \& Arévalo (2007), al realizar una revisión histórica, se encuentra que uno de los primeros autores fue Sócrates. Hace alrededor de 2500 años planteó el método del Diálogo Socrático, que implica cuestionar a las personas para que justifiquen racionalmente sus ideas a partir de su propio conocimiento. Aquí se resalta la importancia de formular preguntas profundas, evaluar procesos intelectuales, presupuestos y creencias y las consecuencias que expresan dichos raciocinios. En palabras del autor, 
"su método es reconocido como una excelente estrategia para desarrollar el pensamiento crítico” (p.41).

Las preguntas definen las tareas, expresan problemas y delimitan asuntos. Impulsan el pensar hacia adelante. Las contestaciones, por otra parte, a menudo indican una pausa en el pensar. (Paul \& Elder, 2002, p. 5). En este planteamiento los investigadores enfatizan en la preponderancia del cuestionamiento y la reflexión sobre problemas sobre las contestaciones o respuestas que anquilosan en avance del conocimiento.

\section{Metodología}

El enfoque metodológico empleado en este trabajo es mixto con mayor énfasis en lo cualitativo. "Este enfoque se selecciona cuando el propósito es examinar la forma en que los individuos perciben y experimentan los fenómenos que los rodean, profundizando en sus puntos de vista, interpretaciones y significados" (Hernández-Sampieri, 2014, p. 358). Lo que se busca en este caso es que los estudiantes tomen conciencia de sus procesos de pensamiento. Sobre este asunto este enfoque resulta propicio para el desarrollo de investigaciones propias del campo de las ciencias sociales y de la educación.

La metodología escogida para la realización de esta investigación fue la Investigación Acción. Según Tamayo y Tamayo (1999), la investigación acción hace parte del enfoque cualitativo y pretende resolver las cuestiones que se derivan comprendidas en un análisis de la realidad de la sociedad y de la destreza práctica del investigador. Este modelo de investigación acepta incorporar en el proceso a los individuos como investigadores eficaces en lugar de verlos como objetos investigados.

Destacando a Elliot (1990), se define la investigación acción como una reflexión sobre las acciones humanas $\mathrm{y}$ las situaciones cotidianas que vive el profesorado, se tiene por objetivo la ampliación de la comprensión de los docentes y de sus situaciones problema. Es decir, busca reestructurar la situación inicial para llegar a una comprensión profunda de los problemas.

\section{Muestra}

Para el objeto de la presente investigación, se tomarán como referencia la totalidad de los estudiantes del curso Sexto B, correspondiente a 29 individuos, en el contexto de la enseñanza de las Ciencias Sociales. De ellos 7 corresponden a hombres y 22 a mujeres.

\section{Categorización en el cuestionario \#1 y Taller \#1 del cine-foro}

Las principales estructuras teóricas que componen un proyecto de investigación son las categorías. En palabras de Bardín (1986), "la categorización es una operación de clasificación de elementos constitutivos de un conjunto por diferenciación, tras la agrupación por género (analogía), a partir de los criterios previamente definidos."( $p$, 90). La categorización es una parte
Destacando a Elliot (1990), se define la investigación acción como una reflexión sobre las acciones humanas y las situaciones cotidianas que vive el profesorado, se tiene por objetivo la ampliación de la comprensión de los docentes y de sus situaciones problema. 
esencial del proceso de análisis, pues de su adecuada elaboración dependerá la calidad de los datos que se logren. Las categorías que han surgido de la investigación documental, en este caso, se desprenden principalmente de los 8 elementos del pensamiento, ya referidos. Las sub-categorías surgen del análisis descriptivo del tema en las respuestas a los cuestionarios y talleres.

Bardin (1986) señala que el proceso de categorización puede efectuarse según criterios semánticos (temas), conceptuales (conceptos), sintácticos (verbos, acciones), léxicos (definiciones). Para el caso de la presente investigación este análisis se realizó desde el aspecto semántico de los temas y sus posibles significados, puesto que, "la semántica es el estudio del sentido de unidades lingüísticas. Por lo tanto, actúa sobre el material principal del análisis de contenido: los significados” ( $p, 33)$.

Análisis de contenido.

En palabras de Bardin(1986), "el análisis de contenido es un conjunto de técnicas de análisis de comunicaciones” (p, 23), en lugar de ser solo un instrumento, el análisis ofrece un amplio abanico de posibilidades de investigación dentro del enfoque cualitativo.

Para Berelson (como se citó en Zapata, 2005), "Dentro del proceso de comunicación, el contenido ocupa una posición central, por el contenido de la comunicación se entiende que los contenidos verbales, musicales, pictóricos, plásticos y gesticulares constituyen la comunicación” (p. 199). El procedimiento de analizar la comunicación representa una gran complejidad, como observa Zapata (2005), pues sus causas y efectos son difíciles de determinar, es necesario por ello describir los rasgos característicos del mensaje analizado. "El análisis de contenido intenta describir las diferentes características del contenido de una comunicación en forma precisa y concisa” (p. 200).

Siguiendo a Bardin (1986), "hay que distinguir entre unidad de registro (lo que se cuenta) y la regla de enumeración (la manera de contar)" (p, 82). Las reglas de enumeración que se utilizaron en el análisis de las unidades de registro fueron:

-Frecuencia de presencia (o de ausencia): aparición de elementos significativos que le den sentido al texto. Si en una determinada serie de resultados aparecen ciertos elementos, éstos serán significativos para la categorización, por ejemplo, si se evalúa el ítem información en uno de los talleres tal vez se espere que los estudiantes describan los elementos pertinentes a la categoría forma de vida de un grupo de personas, dentro de estos elementos pueden aparecer algunas sub-categorías relativas a la política, a la ciencia, a la gastronomía, etc. Mientras más se amplíe el rango y la presencia de interpretaciones diversas, más significativo será para la categorización que se efectúe para dicho ítem. Esta regla fue fundamental para el análisis de los cuestionarios y talleres. La importancia de una unidad de registro se incrementa con su frecuencia de aparición. La medida 
presencial-frecuencial escogida es de tipo simple, lo que quiere decir que, los criterios de la rúbrica de evaluación tienen un mismo peso y se tiene en cuenta la aparición cuantitativa de las unidades de información significativas desde el punto de vista de los elementos del pensamiento.

Para Bardin (1986), “el análisis intenta tomar en consideración la totalidad de un texto para pasarlo por el molinillo de la clasificación y de la enumeración por frecuencia de presencia (o de ausencia) de ítems de sentido" (p, 28). Por ejemplo, la frecuencia en la presencia o ausencia de información en las respuestas a los cuestionarios o la frecuencia con que aparecen los criterios que la rúbrica de evaluación demarca para de cada elemento de pensamiento en los talleres, se puede entender que esta información representa una evidencia por sí misma. En algunos casos la ausencia de elementos (en relación con una expectativa) puede darle sentido. Por poner un caso, omitir el nombre del personaje principal sobre el que se está pidiendo información representa una grave falencia al momento de expresar el pensamiento. En efecto, el estudiante que manifiesta esta omisión o ausencia de información no comprende que su texto debe ser leído e interpretado por otras personas por lo que es necesario que éste se encuentre redactado en forma comprensible.

\section{Unidad De Contexto}

Sirve de unidad de comprensión para codificar la unidad de registro (Bardín,
1986), es superior en tamaño a la unidad de registro y le da significado. Bardin sostiene que las unidades de contexto tiene una especial relevancia cuando el mensaje a analizar es complejo. La unidad de contexto, al ser más amplia, puede corregir el análisis de las otras unidades de contenido. Principalmente se ha considerado como unidad de contexto la coherencia. De este modo, se pueden extraer ciertas hipótesis concernientes a la coherencia o incoherencia, entre la totalidad de las respuestas obtenidas en cuestionarios y talleres, que permiten corroborar algunos efectos de la intervención.

\section{Unidades de análisis.}

En palabras de Hernández-Sampieri (2014), "la recolección de datos sucede en ambientes naturales y cotidianos de los participantes o unidades de análisis” (p, 397). Se trata pues de la vida misma y la experiencia como fuentes primarias de información. Desde luego, la unidad de análisis básica en todo momento son las aportaciones de los propios estudiantes durante todo el proceso y sus interrelaciones con el grupo y el entorno en general. Ahora, ¿Qué tipos de unidades de análisis pueden incluirse en el proceso cualitativo, además de las personas o casos? Lofland et al. (2005, Como se citó en Hernández-Sampieri, 2014, $\mathrm{p}, 397)$ señalan entre otras posibles unidades de análisis:

- Significados: Son definiciones, ideologías o estereotipos que se convierten en referentes lingüísticos para representar una realidad social. 


\section{Unidad de registro.}

"La unidad de registro es la unidad de significación que se ha de codificar es aquello que se pretende evaluar" (Bardín, 1986, p, 90). Éstas pueden ser, según el material o código de que trate; la palabra, la frase, el minuto o cualquier otro segmento de significación. Esta es la unidad de registro elemental para la recolección de los resultados.

- Tema:

El tema es utilizado generalmente como unidades de registro para estudios de motivaciones, de opiniones, de actitudes, de valores, de creencias, de tendencias, etc. "Las respuestas a preguntas abiertas, las conversaciones (conversaciones no directivas o más estructuradas) individuales o de grupo, de encuesta, los protocolos de test,... pueden y a menudo son analizados sobre la base del tema" (Bardín, 1986, p, 80).

\section{Análisis De Datos}

\section{Resultados de la Prueba Diagnóstica}

La prueba diagnóstica consistió en la aplicación de una variación del test Critical Thinking appraisal de Watson y Glaser (Tomado de Di Fabio, 2005), el test usado es una versión reducida de 50 preguntas, distribuidas en 5 test o ítems. La prueba está diseñada para evaluar, por medio del análisis de texto $\mathrm{y}$ de situaciones, algunos elementos del P.C. como son: las inferencias, la evaluación de supuestos, la deducción, la evaluación de argumentos y la interpretación, respectivamente.

Se debe señalar además que la información recogida con esta prueba resultó muy valiosa para la subsiguiente aplicación de la didáctica pues entregó evidencia acerca de los estados iniciales en el manejo de los elementos lógicos del P.C. de la población intervenida.

Tabla 1. Resultados de la Prueba diagnóstica

\begin{tabular}{|l|l|l|l|l|}
\hline $\begin{array}{l}\text { Ítem 1 } \\
\text { Inferencias }\end{array}$ & $\begin{array}{l}\text { Ítem 2 } \\
\text { Reconocer } \\
\text { Supuestos }\end{array}$ & $\begin{array}{l}\text { Ítem 3 } \\
\text { Deducción }\end{array}$ & $\begin{array}{l}\text { Ítem 4 } \\
\text { Interpretación }\end{array}$ & $\begin{array}{l}\text { Ítem 5 } \\
\text { Evaluar } \\
\text { Argumentos }\end{array}$ \\
\hline 3,14 & 11,3 & 10,73 & 11.06 & 11 \\
\hline
\end{tabular}

Esta tabla presenta los datos de la prueba diagnóstica en resultados de 0 a 20 por ítem.

Según la tabla 1 se pudo determinar que la muestra alcanzó un porcentaje de aciertos del $47 \%$ en el total del test. Teniendo en cuenta que la puntuación máxima para cada ítem es de $20 \%$ se obtuvo que el ítem con mejor puntuación general fue Reconocimiento de Supuestos con un 11\% de aciertos; en segundo lugar tenemos al ítem de Interpretación con 
$10.8 \%$, en tercer lugar aparece el ítem de Evaluación de Argumentos con $10.7 \%$, en cuarto lugar está el ítem de Deducción con 10.4 y finalmente el ítem de Inferencias obtuvo un promedio de tan solo 3.14 .

Los anteriores resultados llaman la atención por dos motivos, en primer lugar, porque en casi todos los ítems se alcanzan porcentajes de acierto superiores al $50 \%$, esto es interesante porque se alcanzan puntuaciones ligeramente por encima de la media, considerando que la prueba en estos ítems en particular se decide entre un $50 / 50$ en vista de que solo hay dos opciones, resulta satisfactorio el hecho de que en esta etapa inicial se alcancen estos promedios. En segundo lugar, llama la atención el resultado del ítem de Inferencias, este ítem es diferente de los otros pues presenta cinco opciones diferentes de respuesta, obligando al estudiante a matizar el grado de validez de cada inferencia; esto obviamente complica la capacidad de lograr aciertos en la mayoría de los estudiantes de la muestra.

Este ítem solo obtuvo un porcentaje de aciertos equivalente al 3.14\% sobre un total de $20 \%$. Es obvio que los resultados de este ítem influyeron negativamente sobre los puntajes totales de la prueba que cayeron hasta el 47\%.

Resulta también satisfactorio el hecho de que se encuentran estudiantes con puntajes relativamente altos en la prueba general. Cinco estudiantes de la muestra lograron puntajes de acierto por encima de $60 \%$, otros 5 estudiantes lograron puntajes entre 50\% y 60\%; 16 estudiantes con resultados que oscilan entre el 40\% y 50\% de aciertos y solo 3 estudiantes con promedios inferiores al $40 \%$.

\section{Resultados cuestionario \#1}

La primera prueba abierta que se ha aplicado consiste en un cuestionario de ocho preguntas inspirado en la obra de Paul \& Elder (2003, 2005), que permiten evaluar ocho ítems diferentes que se corresponden con los elementos del pensamiento que se han propuesto en esta investigación como componentes básicos del P.C. a saber: problema, propósito, información, conceptos, suposiciones, conclusiones, consecuencias y punto de vista, respectivamente.

Este cuestionario se diseñó con el fin de obtener información, datos e hipótesis sobre el primer ciclo de cine-foro; el cuestionario se centra en el análisis de un personaje de la película "Los Croods". Se pidió a los estudiantes que aplicaran los elementos de P.C. al personaje que ellos consideraban que es el principal, basando su apreciación, en buena medida, en los productos surgidos del conversatorio que se realizó con posterioridad a la proyección de la película. Para evaluar el anterior instrumento se hizo uso de una rúbrica diseñada con el propósito de cuantificar la información que resultara de los cuestionarios y talleres que se realizaran en cada uno de los ciclos de películas.

La rúbrica tiene una escala de calificación que va de 0 a 3 para cada
Este cuestionario se diseñó con el fin de obtener información, datos e hipótesis sobre el primer ciclo de cine-foro; el cuestionario se centra en el análisis de un personaje de la película "Los Croods". 
ítem, estos valores representan cuatro criterios de evaluación que se refieren a grados o matices en la capacidad del estudiante para asimilar y expresar un pensamiento correctamente. Estos criterios obedecen a caracterizaciones propias de cada elemento del pensamiento que han aflorado en la investigación teórica y documental. Los estándares intelectuales descritos en la obra de Paul \& Elder (2003,2005) han surgido como los patrones de medida más adecuados para la evaluación, en particular en lo que se refiere a la claridad y a la precisión evidenciada para asimilar y expresar el pensamiento propio y ajeno.

De este modo tenemos como primer nivel de respuesta la nulidad o completo sinsentido en una respuesta. Sucede cuando el estudiante no ha contestado a la pregunta o ha contestado algo que no tiene relación alguna con la cuestión requerida. Este criterio corresponde al valor de 0 , Nulo.

El segundo nivel de respuesta tiene que ver con un grado mínimo de comprensión con respecto al concepto en cuestión aunque sin demasiada claridad o precisión en la expresión del pensamiento. Este criterio corresponde al valor de 1 , Iniciado.

El tercer nivel de respuesta determina que el estudiante tiene cierta claridad sobre el concepto evaluado, pues puede expresar su pensamiento claramente, no obstante, sus respuestas no son realmente atinadas o precisas desde el aspecto crítico. Estecriterio corresponde a un valor de 2, Satisfactorio.
El mayor nivel de respuesta es el cuarto, aquí el estudiante evidencia una comprensión óptima del concepto y lo aplica con claridad, además llega a conclusiones significativas desde la perspectiva crítica. En este punto el estudiante demuestra que ha interiorizado el concepto y lo maneja con fluidez. Este criterio corresponde a un valor de 3, Excelente.

Al aplicar la rúbrica a los datos obtenidos en la prueba de análisis del primer ciclo de cine-foro se registraron resultados esclarecedores para la realización de los subsiguientes ciclos programados en el proyecto de investigación.

En primer lugar, se pudo determinar que existe un grado de coherencia suficiente entre los resultados obtenidos en la prueba diagnóstica y la prueba abierta del cuestionario. Aunque hay notables diferencias en algunos aspectos, se puede decir en general que existen puntos de conexión que en cierto modo validan sus resultados mutuamente; por ejemplo, los resultados generales en la prueba cerrada rondaban el 47\% de aciertos, en el caso de la prueba abierta rondaban el 13.68 de aciertos sobre 24 puntos posibles, es decir, el 57\%, teniendo en cuenta que entre ambas pruebas medió el primer ciclo del cine-foro pues era de esperarse que hubiera algunos avances en la comprensión de los conceptos analizados; además, la media de aciertos por respuesta registrada fue de 1.69 sobre 3, lo cual está ligeramente por encima de los resultados de la prueba cerrada. Esto es tranquilizante porque 
los resultados no se contradicen (salvo en contadas excepciones) y concede una mayor seguridad en la aplicación de dichos instrumentos.

En segundo lugar, resultó claro que hay ciertos ítems evaluados que distorsionan los resultados generales. En el caso de la prueba cerrada este ítem correspondió a las inferencias y deducciones, en el caso de la prueba abierta este ítem correspondió a información puesto que los demás ítems tuvieron promedios de aciertos mayores. El ítem de información, sin embargo, no se evaluó en la prueba cerrada debido a que este ítem está enfocado a rasgos pertinentes a los contenidos temáticos de la Ciencias Sociales, que es la asignatura en la que se contextualiza el proyecto.

Tabla 2. Resultados del cuestionario \#1

\begin{tabular}{|l|l|l|l|l|l|l|l|}
\hline Ítem 1 & Ítem 2 & Ítem 3 & Ítem 4 & Ítem 5 & Ítem 6 & Ítem 7 & Ítem 8 \\
Propósito & Problema & Informa & Concepto & Supuesto & Inferencia & Consecue & P. vista \\
\hline 2,16 & 2,08 & 0,64 & 1,84 & 1,8 & 1,64 & 1,64 & 1,84 \\
\hline
\end{tabular}

Esta tabla contiene los elementos de pensamiento y sus resultados en el cuestionario \#1 con puntajes de 0 a 3 por ítem.

El cuestionario tiene una puntuación máxima de 24 puntos y cada respuesta tiene una puntuación máxima de 3 puntos. El ítem 3, correspondiente al concepto Información obtuvo solo 0.64 sobre 3 puntos. Como muestra la tabla 2 , este ítem fue el único que estuvo por debajo de 1 en el promedio general. Excluyendo este puntaje tan bajo se ven promedios de 2.16, 2.08, 1.84, 1.80, $1.64,1.64$ y 1.84 respectivamente en los otros ítems.
Por otro lado, los ítems 1 y 2, correspondientes a los conceptos Propósito y Problema, muestran promedios por encima de 2, lo cual es muy alentador pues han sido los conceptos más analizados durante el primer foro junto con el concepto de inferencia (ítem 6) que mostró también un progreso respecto de los resultados obtenidos en la prueba cerrada.

Como se había explicado antes, a los estudiantes se les pidió identificar al personaje principal y aplicar el concepto Problema. De esta elección del personaje aparecieron ejes temáticos particulares que pudieron ser desglosados para su posterior análisis. 
Tabla 3. Categorización del cuestionario \#1

\begin{tabular}{|l|l|}
\hline Personaje & $\begin{array}{l}\text { Ejes temáticos de las problemáticas y } \\
\text { categorías }\end{array}$ \\
\hline Grug & $\begin{array}{l}\text { Miedo al exterior, La muerte (M): 3 } \\
\text { estudiantes } \\
\text { Ser inteligente (I): 1 estudiante }\end{array}$ \\
\hline Eep & Libertad, aventura (L): 4 estudiantes \\
\hline Temática-Protagonista & $\begin{array}{l}\text { Relación padre e hija (RPH): 9 } \\
\text { estudiantes }\end{array}$ \\
\hline Todos & $\begin{array}{l}\text { Supervivencia, la destrucción de su } \\
\text { mundo, Buscarcomida(S): 4 estudiantes }\end{array}$ \\
\hline Chico: & Ganarse la confianza, (C): 1 estudiante \\
\hline
\end{tabular}

La tabla muestra las categorías y subcategorías que emergieron en el ítem 2. Problema en el cuestionario \#1.

Lo más significativo de estos resultados mostrados en la tabla 3, radica en el hecho de que muchos estudiantes simplemente omitieron explicar en su respuesta cuál era el personaje sobre el que estaban hablando. Asumieron que esto era obvio y no se molestaron en poner un sujeto a los predicados que escribían. Esto viola los estándares propios para evaluar el P.C. porque si el estudiante no es consciente de que el objetivo de expresarse es que los otros lo entiendan pues no se puede ser claro ni preciso. No obstante, esta dificultad, en virtud de la posible coherencia en las respuestas de cada estudiante, puede inferirse el personaje en cuestión, permitiendo al menos suponer si el estudiante comprende el concepto requerido, pues pueden asociarse problemáticas específicas a personajes específicos.
Los estudiantes que identificaron a Grug (el padre) como protagonista señalaron como sus problemas principales "el miedo al exterior", "a la muerte" y "la inteligencia". E292 (estudiante \#29), contestó que el problema del personaje era el miedo al exterior, aunque no especificó que este personaje era Grug, la coherencia con el resto de sus respuestas permitió establecer que hay una comprensión suficiente del concepto pero que no lo expresa con claridad y precisión, su nota fue de 2, satisfactorio. La respuesta de E28 "el miedo a la muerte" no expresa más que una comprensión muy vaga pues el miedo a la muerte no es un atributo que caracterice al personaje, es un temor universal pero que no lo describe, su calificación fue de 1 , Iniciado. E21 contestó que "el problema de Grug es la inteligencia”, esta respuesta evidencia una incomprensión del concepto, ya que en todo caso el problema sería la falta de inteligencia, o que la inteligencia de alguien más 
perjudica a Grug, como sea, la respuesta carece de sentido, su calificación fue de 0, Nulo.

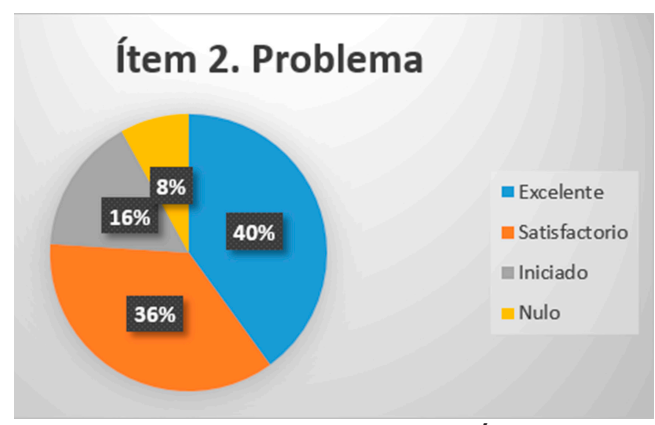

Figura 1. Resultados del Ítem 2.

Problema, en el Cuestionario \#1

La figura 1, se explica porque 10 estudiantes obtuvieron en este ítem la calificación máxima de 3 puntos por lo que tuvieron que cumplir con los tres criterios de evaluación propios para merecer esta nota, a saber:

- Posee una clara comprensión de la pregunta-problema principal que tratan de responder, del problema que tratan de resolver o del asunto que tratan de atender.

- Formula sus preguntas-problemas de manera clara y precisa, reconociendo cuando se trata de un problema complejo.

- Además, los estudiantes que piensan críticamente, analizan y evalúan el uso de preguntas y problemas con el pensamiento de otros.

Las personas que hablaron de una temática protagonista pudieron dar cuenta de una problemática central en la película. Se trata de la relación padre (Grug) e hija (Eep), realmente E14,E15 y E27 entre otros quisieron decir que reconocían a Eep como protagonista e identificaban la relación con el padre como su problemática particular, esto se entiende por el contexto de las demás respuestas. En general, las calificaciones logradas en este ítem por las personas que contestaron en este sentido fueron de 3 puntos, puesto que identificar una temática central con claridad requiere de cierto grado de comprensión del concepto.

E8 contesta que "la problemática del protagonista es la libertad, a juzgar por el contexto se refiere a Eep”. Solo logró 1 punto pues su respuesta es tan vaga que no permite saber exactamente por qué la libertad es un problema, tal vez quiso decir que la falta de libertad de Eep es un problema.

\section{Resultados del Taller Ciclo \#1:}

El taller correspondiente al Ciclo \#1 del Cine-Foro fue un instrumento que se usó para evaluar los avances logrados al finalizar este primer ciclo. Cabe recordar que la película proyectada fue Los Croods, esta taller, que se aplicó a esta película tras la realización del Foro- conversatorio, arrojó resultados preliminares muy útiles.

El taller por su parte es mucho más extenso que aquella prueba pero evalúa exactamente los mismos 8 ítems, usando para su calificación la misma rúbrica. En este caso cada ítem consistió de tres puntos que se encuentran interrelacionados en mayor o menor medida, lo cual otorga mucha más profundidad al momento de determinar si el concepto 
analizado ha sido interiorizado por el estudiante. Por consiguiente, la puntuación máxima por ítem es de 3 y la puntuación máxima de la prueba es de 24 (al igual que sucedía con la prueba de análisis), calificados bajo los criterios consignados en la rúbrica: sentido crítico, claridad y precisión.

Además, las unidades de análisis (tema) y las unidades de contexto (coherencia) y la unidad de regla de enumeración (frecuencial-presencial), siguen siendo la base para la configuración de las subcategorías de análisis. En esta prueba, no obstante, no es posible guiarse solo por un personaje para realizar un análisis más detallado, de manera que se hace imprescindible en muchas ocasiones recurrir a las propias escenas de la película como referente inmediato de análisis.

Los datos arrojados por este taller muestran un ligero incremento en los índices de acierto con respecto a los obtenidos en el cuestionario \#1. En el caso de la primera prueba abierta rondaron el 13.68 de aciertos sobre 24 puntos posibles, es decir, el 57\%, en el caso de la segunda prueba abierta, es decir, el taller, se obtuvieron resultados de 14,3 , lo que es equivalente a un $60 \%$. El promedio de aciertos por ítem pasó de 1,69 a 1.76 .

Tabla 4. Resultados del Taller \#1

\begin{tabular}{|l|l|l|c|l|c|c|c|}
\hline Ítem 1 & Ítem 2 & Ítem 3 & Ítem 4 & Ítem 5 & Ítem 6 & Ítem 7 & Ítem 8 \\
Propósito & Problema & informa & Concepto & Supuesto & Inferencia & Consecue & P.vista \\
\hline 2,46 & 2,0 & 1.53 & 1,34 & 1,53 & 1,69 & 2.19 & 1,46 \\
\hline
\end{tabular}

Esta tabla contiene los elementos de pensamiento y sus resultados en el taller \#1 en puntajes de 0 a 3.

Estos resultados que aparecen en la tabla 4, permiten corroborar un avance paulatino en ciertos aspectos; por ejemplo, el ítem que obtuvo la peor calificación en el cuestionario \# 1 fue el Ítem 3 correspondiente a Información con 0.64; esta vez se obtuvo un puntaje de 1.53 en este ítem; de hecho, ningún ítem estuvo por debajo de 1 en el promedio.
De este modo resultó que los ítems con mejor puntuación fueron los que corresponden a Propósito (ítem 1), a Problema (ítem 2) y a Consecuencia (ítem 7) con 2,46, 2,07 y 2.19 respectivamente. Estos ítems estuvieron por encima de 2 en promedio, lo cual se puede considerar un puntaje muy alto.

Con 1.53 tuvimos los ítems Información (ítem 3) y Supuestos (item 5), ligeramente por encima de la media; Inferencias (ítem 6) obtuvo un 
puntaje de 1.69 , siguiendo la tendencia positiva mostrada en el cuestionario. Finalmente solo un ítem estuvo por debajo de la media, Concepto (ítem 4) con 1.34.

Al comparar las dos pruebas aplicadas para el primer ciclo se podría esperar cierta coherencia entre ellas en vista de que los talleres se diseñaron a partir del cuestionario; en efecto, esta coherencia se encuentra evidenciada en que entre los puntajes más altos se encuentran los Ítems 1 y 2 que también fueron los mejores en el cuestionario. Mientras que los demás ítems obtuvieron resultados bastante parecidos en ambas pruebas, aunque con dos importantes excepciones: Consecuencias (ítem 7) y Conceptos (ítem 4).

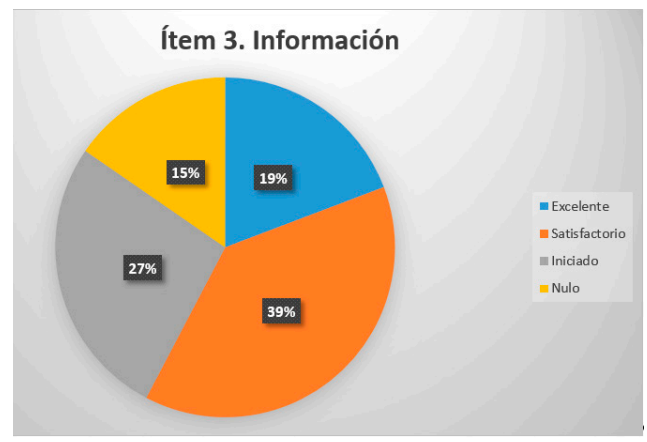

Figura 2. Resultados del Ítem 3. Información en el taller \#1.

Este ítem mostró importantes avances en los niveles de acierto de este taller, como señala la figura 2. En el cuestionario \#1 este fue el ítem con peor calificación (0.64) y aquí fue de 1.53. Nuevamente, las actividades en el aula parecen soportar los datos alcanzados en la evaluación.

La comprensión necesaria del concepto es mucho más amplia en el taller que la que se pide en el cuestionario, pues en aquella primera evaluación solo se tenía el foro como referente. Aunque el foro intentó por medio del diálogo encaminar el análisis hacia todos los ítems, solamente algunos de ellos pudieron ser claramente abordados.

En cambio, en este momento de la secuencia, cuando ya se han aplicado las actividades propuestas para el primer ciclo, la intervención debería empezar a mostrar sus efectos. Las actividades posteriores, acompañadas de las guías pertinentes a las temáticas propias de las Ciencias Sociales (en este caso "la edad de piedra") (Galindo, et al., 2007), definitivamente tenían que hacer una diferencia notable en lo que refiere al ítem Información en particular. En efecto, este ítem requería tener información confiable con la cual contrastar la información presentada en la película. Para muchos estudiantes este ítem pareció más evidente y claro pues tenían información fidedigna con la cual apoyar su opinión.

Esto puede explicar que 4 estudiantes lograran la nota máxima y que 11 lograran la nota de 2 puntos; por lo tanto, 15 estudiantes, que corresponden a más de la mitad de la muestra, desarrollaron una mayor claridad en este concepto. Por otro lado, 6 personas tuvieron nota de 1 y 8 de ellos no tuvieron nota; este índice de vaguedad o nulidad en el concepto siguió siendo alto. El concepto Información era más desafiante que otros pues obligaba al estudiante a investigar y consultar los datos históricos involucrados en cada uno de los puntos de este 
ítem y relacionarlos con su propio conocimiento al respecto.

El ítem escogido para ejemplificar el proceso de categorización en este taller \#1 es el ítem 3. Información. Se propone recoger datos acerca de la correcta apropiación de la información emergente en la película. La prueba presenta 3 puntos para este ítem. Cada punto consiste en tres preguntas compuestas, es decir que cada pregunta se compone de hecho de dos preguntas: para el análisis se consideraron los argumentos expuestos por los estudiantes en sus respuestas. Si la respuesta es clara, precisa y coherente entra en una de las subcategorías de análisis y el punto se considera válido, de lo contrario no se tiene en cuenta para la categorización. De este modo surgió la siguiente categorización:

Tabla 5. Categorización taller \#1 -punto a.

\begin{tabular}{|l|l|}
\hline \multicolumn{1}{|c|}{ Son consecuentes } & \multicolumn{1}{|c|}{ No son consecuentes } \\
\hline -forma de vida (FV): 14 estudiantes & -fauna y flora (FF): 3 estudiantes \\
-descubrimientos (D): 5 estudiantes & -ciencia ficción (CF): 4 estudiantes \\
-teoría evolutiva (TE): 3 estudiantes & -evolución rápida (ER): 1 \\
& estudiante \\
& -anatomía (A) 1 estudiante \\
\hline
\end{tabular}

Esta tabla presenta las categorías y sub- con la información presentada en categorías que surgieron en el ítem 3. las guías con respecto a la época Información, punto a., del taller \#1. prehistórica ¿Son consecuentes las dos Plantea la pregunta: Compara la información presentada en la película versiones?, ¿en qué se asemejan y en qué se distinguen?

Tabla 6. Categorización taller \#1 -punto b.

\begin{tabular}{|l|l|}
\hline Es confiable & No es confiable \\
\hline -forma de vida (FV): 10 estudiantes & -ciencia ficción (CF): 3 estudiantes \\
-descubrimientos (D): 4 estudiantes & -fauna y flora (FF): 2 estudiantes \\
- teoría evolutiva (TE): 2 estudiantes & \\
\hline
\end{tabular}


Esta tabla presenta las categorías y subcategorías que surgieron en el ítem 3. Información, punto b., del taller \#1.
Plantea la pregunta: ¿La película presenta una información confiable y apegada a las hipótesis científicas? Explica por qué.

Tabla 7. Categorización taller \#1 -punto c.

\begin{tabular}{|l|l|}
\hline Desarrollo de la familia Croods & Evolución humana \\
\hline -descubrimiento (D): 7 estudiantes & -Origen (O): 1 estudiante \\
-Experiencia Vital (EV): 2 estudiantes & -Evolución (E): 7 estudiantes \\
& -Supervivencia (S): 3 estudiantes \\
\hline
\end{tabular}

Esta tabla presenta las categorías y subcategorías que surgieron en el ítem 3. Información,

El punto c., plantea la pregunta: ¿Puedes describir la transformación que sufre la familia Croods a lo largo de la película, contrastar estos dos momentos con las hipótesis actuales sobre el desarrollo y evolución de la humanidad?

Las tablas 5,6 y 7, evidencian que los estudiantes que comprendieron el concepto y lograron acertar en este punto ofrecieron argumentos $\mathrm{u}$ opiniones que contenían temáticas directamente relacionadas con $L a$ Edad De Piedra. Para lograr el acierto era necesario que el estudiante pudiera explicar clara y precisamente dicha información y que además pudiera utilizarla de manera práctica al contrastar lo que ve en la película con la información que él mismo posee.

Así por ejemplo: las respuestas de E1, E9, E14, E22, y E25 fueron: "a-no son consecuentes, había depredadores, cavernícolas, descubrieron el fuego, la película es de ciencia ficción.

b- muestra como cazar, como tenía que convivir entre ellos y como tenían que sobrevivir.

c- empezaron a separarse y a pensar más”

Aunque el punto a y el punto b no están muy claramente redactados dejan entrever las categorías Forma De Vida, Descubrimiento, Supervivencia, como aspectos en los que el documento fílmico es consecuente con los hechos históricos analizados en las guías complementarias y en las actividades de aula. Por otro lado, reconoce que la obra es de Ciencia Ficción y en este sentido no puede ser plenamente consecuente. La calificación de estos estudiantes fue de 2, puesto que la respuesta al punto c es muy ambigua y no responde satisfactoriamente a lo que se pide; aquí no se establece la relación entre el desarrollo particular de los
Aunque el punto a y el punto $b$ no están muy claramente redactados dejan entrever las categorías Forma De Vida, Descubrimiento, Supervivencia, como aspectos en los que el documento fílmico es consecuente con los hechos históricos analizados en las guías complementarias y en las actividades de aula. 
Croods con el desarrollo evolutivo de la humanidad en general.

Las respuestas de E28 a este ítem fueron:

" $a$ - Si son cosencuentes. Ya que ellos batallan por la comida y viven en cuevas y eran cavernícolas. Que ellos se comunicaban por medios diversos y se distingune en lo rápido

b-yo creo que sí. Porque la epoca prebistoria es como embase a la pelicula

c-de que al cambiar de origen se ivan a crear nuevas cosas"

Este estudiante argumenta que la película es consecuente con los hechos históricos, señala aspectos relativos a la subcategoría Forma De Vida y además agrega la subcategoría Evolución Rápida como un aspecto no consecuente. $\mathrm{Su}$ calificación de 2 es debida a que las respuestas están planteadas de una manera clara, coherente y suficiente; los puntos a, b y c permiten contrastar una información propia del estudiante con información provista por la película. El punto c es tal vez el menos claro señala los temas Origen Y Descubrimiento que son esenciales en la subcategoría Evolución Humana.

Las respuestas de E18 fueron:

"a- si son consecuentes las dos versiones se asemejan en que ambos eran cabernicolas y se distiguen en la forma de su rostro en sus dientes que eran grandes.

b- la pelicula se asemeja en gran parte a las cosas científicas pero pucieron mucha ciencia ficcion c- la transformacion empiezan cuando abandonan su cueva y luego utiliza zapato $y$ el fuego, el fuego fue muy util para ellos como cocinar los alimentos y protección. Que en estos días nos sirven para lo mismo."

E18 obtuvo la calificación máxima pues demostró coherencia, claridad y precisión con la información. Aporta la temática Forma de vida como razón de consecuencia en la información y aporta las temáticas Anatomía y Ciencia Ficción como razones de inconsecuencia y de falta de confiabilidad de la información. El punto c está correctamente resuelto pues hace una enumeración de aspectos propios de la temática Descubrimiento y Forma de vida relacionándolos con la Evolución Humana hasta nuestros días.

\section{Conclusiones}

La investigación ofreció abundantes frutos en cuanto a ideas y perspectivas para futuros proyectos. Los Supuestos o hipótesis iniciales se vieron respaldados por los resultados finales. Estos supuestos consistían principalmente en la necesidad de desarrollar habilidades de pensamiento crítico a través de una estrategia didáctica. El supuesto derivado de aquél es que el Cine-Foro resulta ser un espacio óptimo para el desarrollo de dichas habilidades. El simple hecho de que cada nuevo ciclo de Cine-Foro contribuyera de manera tan notable a tematizar el Pensamiento y desglosarlo en sus partes constitutivas basta para afirmar la validez de la estrategia escogida.

Teniendo estos dos puntos de partida surgió la pregunta que orientó toda 
la investigación. $\mathrm{Y}$ en respuesta a ella, se puede afirmar que el CineForo ha contribuido a empoderar el pensamiento Crítico de los estudiantes de $6 \mathrm{~B}$ de las siguientes formas:

- Permitiéndoles reconocer con cierto detalle elementos de su pensamiento.

- Ejercitando su capacidad crítica frente a situaciones que se les presentan.

- Demostrándoles que las películas que cotidianamente ven pueden ser analizadas críticamente para enriquecer su significado.

Estas respuestas apuntan a la consecución de los objetivos planteados inicialmente en el diseño de la estrategia. Especialmente, el objetivo general que consistió en desarrollar procesos de pensamiento crítico a partir del Cine-foro como estrategia didáctica en los estudiantes de Sexto B grado de la Escuela Normal Superior Antonia Santos (ENSAS), indudablemente se ha conseguido. Estos procesos han abarcado un amplio espectro de conceptos inherentes al pensamiento humano, tarea que para nada es fácil.
En vista del escaso tiempo disponible y a otras dificultades aparejadas al desarrollo de un proyecto de mayor envergadura fue necesario potenciar el tiempo disponible para maximizar la efectividad de la didáctica. En consecuencia, gran parte del éxito de la estrategia tuvo que ver con formar ciertos estándares del pensamiento en un corto espacio de tiempo.

En este sentido, la propuesta ha contribuido a lograr el objetivo general planteado. La estrategia diseñada y aplicada ofreció resultados consistentes y sólidos que condujeron a realizar a su vez el objetivo general.

Acerca de fomentar el uso de espacios y didácticas alternativas para la enseñanza de la asignatura de Ciencias Sociales, se puede decir que la estrategia siempre consideró los contenidos temáticos de la asignatura y respetó los ordenamientos propios de los Derechos Básicos de Aprendizaje (DBA). Los talleres, especialmente, resultaron muy fructíferos para constatar la apropiación de la información que propiciaban las películas y las actividades de aula por la calidad de las subcategorías que fueron emergiendo.

\section{Referencias}

Bardin, L. (1986). Análisis de contenido. (S.L) Ediciones Akal.

Cañizalez, N., \& Pulido, O. (2015). Infancia, una experiencia filosófica en el cine.PraxisษSaber, 6(11),245-262.https://doi.org/10.19053/22160159.3583

Carrasco, J. (2004). Una Didáctica para hoy. Cómo enseñar mejor. Madrid: Ed. Rialp 
Di Fabio, H. (2005). Competencias para la Comprensión de Textos y el Pensamiento Crítico en Nivel Medio y Universitario. Tesis de doctorado. Cuyo: Universidad Nacional de Cuyo.

Elder, L. \& Paul, R. (2002). Un bolsilibro sobre el arte de formular preguntas esenciales. Basado en conceptos de pensamiento crítico y principios socráticos. Traducción con autorización de la Fundación para el Pensamiento Crítico. Consultado el 15 de junio de 2016 en http://www.criticalthinking.org/ resources/PDF/SPAskingQuestions.pdf

Elliot, J. (1990). La investigación-acción en educación. (s.1): Morata.

Galindo, L., Muñoz, M., \& Neira, A. (2007). Nuevas Ciencias Sociales 6. Bogotá: Ed. Santillana

Hernández-Sampieri F. (2014). Metodología de la investigación. México: Mac Graw-Hill. Interamericana ed.

Torres, G., Guzmán, G., \& Arévalo, E. (2007) Manifestaciones individuales de pensamiento crítico en los estudiantes de la Universidad Antonio Nariño, Ibagué. Manizales: Centro de Estudios Avanzados en Niñez y Juventud alianza de la Universidad de Manizales y el CINDE.

Herrero, J. (2016). Elementos del pensamiento crítico, (s.l): Marcial Pons. Ediciones Jurídicas y Sociales.

Paul, R., Binker, A., Jensen, K., \& Kreklau, H. (1990). Critical Thinking Handbook: 4th to 6th Grades. A Guide for Remodelling Lesson-Plans in Language Arts, Social Studies, \& Science. Foundation for Critical Thinking. Sonoma State University.

Paul, R., \& Elder, L. (2003). La mini-guía para el pensamiento crítico conceptos y herramientas. Obtenido de Fundación para el Pensamiento Crítico: https:// www.criticalthinking.org/resources/PDF/SP-ConceptsandTools.pdf

Paul, R., \& Elder, L. (2005). Una guía para los educadores en estándares de competencia para el pensamiento crítico. Fundación para el Pensamiento Crítico www. criticalthinking.org

Presidencia de la República de Colombia. (1994). Ley General de educación 115. Art. Art. 3.

Tamayo y Tamayo, M. (1999). El Proceso de la Investigación Científica. México: Editorial Limusa.

Zapata, O. (2005). La aventura del pensamiento crítico. Herramientas para elaborar tesis e investigaciones socio-educativas. México: ed. Pax México. 